\title{
Position Averaged Convergent Beam Electron Diffraction
}

\author{
J. M. LeBeau,* S. D. Findlay,** L. J. Allen, ${ }^{* * *}$ and S. Stemmer*
}

* Materials Department, University of California, Santa Barbara, CA 93106-5050, U.S.A.

** Institute of Engineering Innovation, School of Engineering, The University of Tokyo, Tokyo, 113-8656, Japan

*** School of Physics, University of Melbourne, Victoria 3010, Australia

Accurate determination of TEM sample thicknesses is critical for many applications, such as quantitative comparisons between experimental STEM images and simulations [1]. Many of the commonly used techniques are either not suitable over a wide thickness range, or are influenced by parameters that are difficult to determine independently, such as surface layers in low-loss EELS. In other cases, thickness measurement techniques require changing the electron optical or imaging conditions. For example, the two-beam convergent beam electron diffraction (CBED) technique [2] requires the operator to change the experimental conditions by tilting to two-beam conditions, and the sample region must be thicker than those for typical high-resolution imaging $(>50 \mathrm{~nm})$. Here we present a new technique, position averaged CBED (PACBED), which, in conjunction with simulations, can be used to measure local sample thicknesses between 1 and $100 \mathrm{~nm}$ without changing from atomic resolution STEM imaging conditions.

An FEI Titan 80-300 kV S/TEM operating at $300 \mathrm{kV}$ was used for CBED pattern acquisition with a Gatan Ultrascan 1000 CCD camera. The STEM probe was set to a convergence semiangle of $9.4 \mathrm{mrad}$. The large convergence angle forms Kossel patterns, which are highly dependent upon probe position relative to the unit cell. To nullify these effects, PACBED patterns are acquired while scanning the electron probe across the image area. In this study experimental PACBED patterns from $\mathrm{SrTiO}_{3}$ and $\mathrm{PbWO}_{4}$ crystals are compared with absorptive Bloch wave and multislice simulations. Figure 1 shows that the patterns are very sensitive to thickness changes that allow direct visual measurement of thickness. During a microscope session, the pattern change with thickness is sufficient to allow for thickness determination from a pre-calculated set of simulations to within about $\pm 2-4 \mathrm{~nm}$.

In addition to thickness measurements, these patterns also provide direct feedback to the operator about how well the sample is aligned to the incident probe. Specimen tilts result in a pattern that is no longer concentric to the central disk as shown in Figure 2. Tilts as small as $0.5 \mathrm{mrad}$ are observable and can be measured.

[1] J. M. LeBeau, S. D. Findlay, L. J. Allen, S. Stemmer, Phys. Rev. Lett. 100, 206101 (2008).

[2] P. M. Kelly, et al., Physica Status Solidi (a), 31, 771, (1975).

[3] S. S. and J. M. L. acknowledge the NSF for support of this research (grant number DMR0804631). L. J. A. acknowledges support by the Australian Research Council. S. D. F. is supported as a Japan Society for Promotion of Science (JSPS) fellow. 

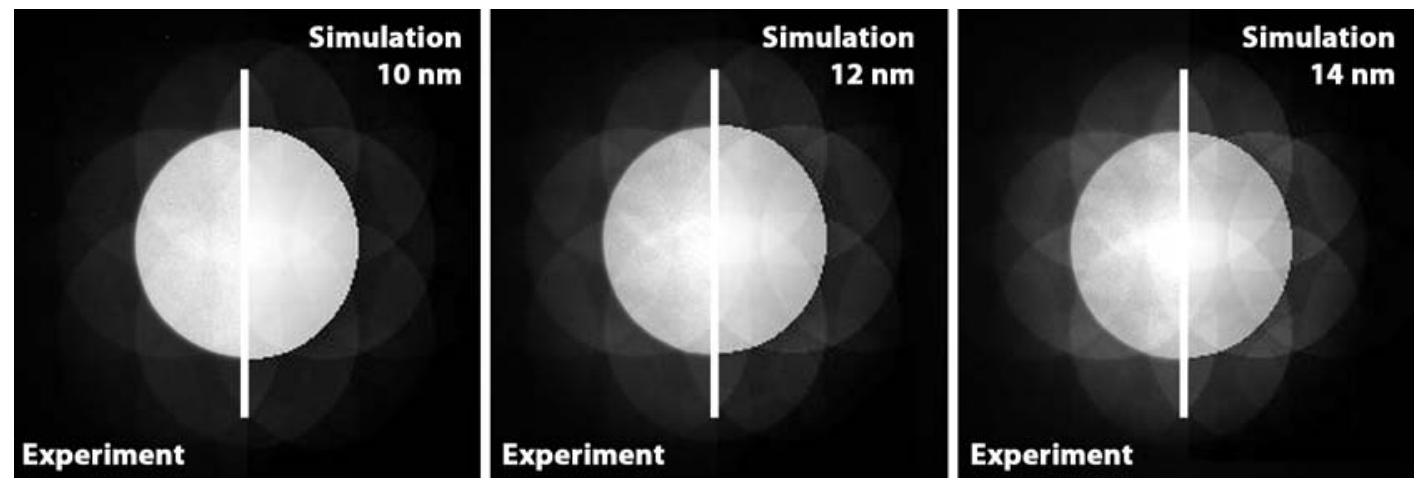

FIG. 1. Experimental (left panels) and simulated (right panels) PACBED for different thicknesses (see labels) of a $\mathrm{PbWO}_{4}$ single crystal observed down the [100] zone axis of the tetragonal scheelite structure.
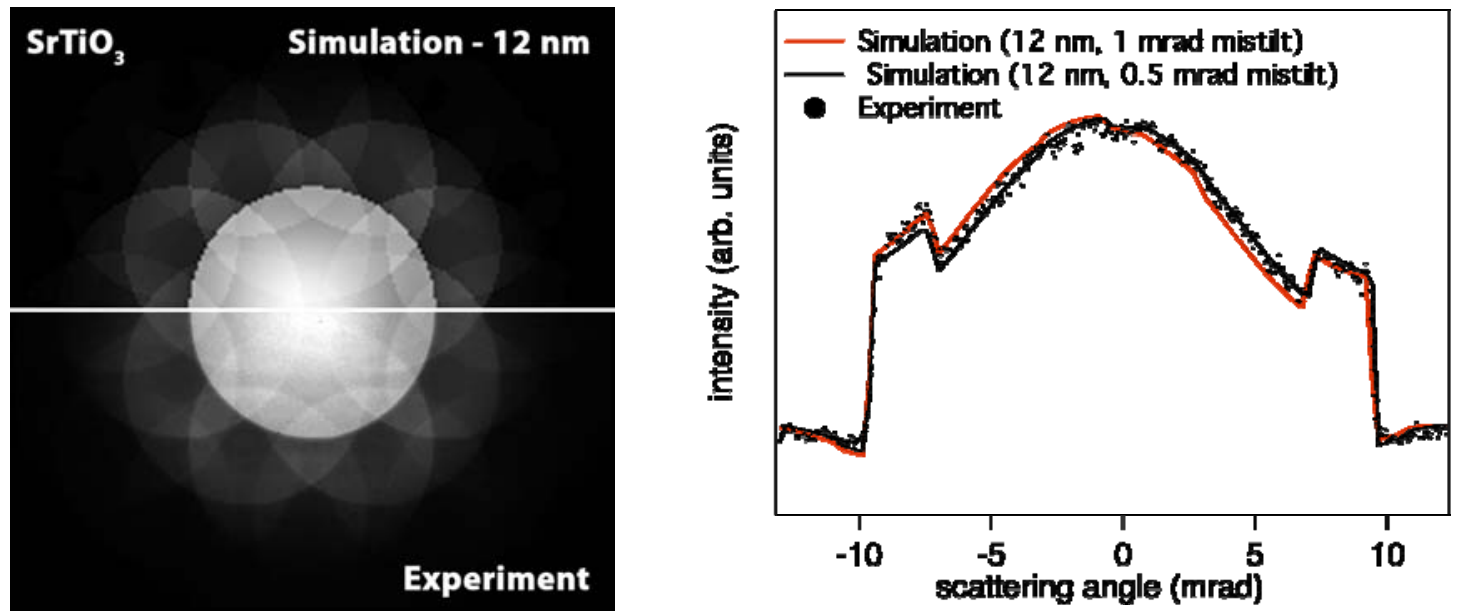

FIG. 2. (a) Experiment (bottom panel) and simulation (top panel) of the effects of $1 \mathrm{mrad}$ tilt on PACBED patterns for a $\mathrm{SrTiO}_{3}$ single crystal ([001] zone axis). (b) Line profile parallel to [100] of the PACBED patterns in (a) for experiment (symbols) and simulations (solid lines). Note that even a small mistilt of $0.5 \mathrm{mrad}$ generates clear asymmetry of the profile. 